\title{
Length measurements in digital images by using an anchored discrete convolution algorithm
}

\author{
A. Suhadolnik, J. Petrišič \& F. Kosel \\ University of Ljubljana, Faculty of Mechanical Engineering, Slovenia
}

\begin{abstract}
A digital image could be used as a source of data for various dimensional measurements. After acquiring the digital image of the measured object, the object contour is determined. The contour is composed of a sequence of distinct pixels which contain centre points. This is a representation of a digital curve or digital arc from which the object length or the perimeter could be determined. In order to calculate the approximate length of the object, we introduce a new algorithm called an anchored discrete convolution. The algorithm is based on a discrete convolution by using different convolution masks and points anchored in the pixels. Unfortunately, the use of an ordinary convolution distorts the curve shape and introduces large errors in length calculation. In our case the anchoring limits the point shifting into the pixel area during the calculation of the curve length. In this paper different convolution masks are used on an experimental digital curve in order to evaluate the presented algorithm as a measuring tool. In addition, different real objects were optically scanned and the perimeters of the objects were determined from the acquired images.
\end{abstract}

Keywords: anchored discrete convolution, digital image, length measurement.

\section{Introduction}

Several different geometrical measurements have digital images as a measuring data source. First the objects are scanned with digital receivers (digital cameras, scanners, CCD arrays etc.) which acquire real images and transform them into digital grey level images. The basic elements of two dimensional digital grey level images are pixels. The digital image data are processed with some 
threshold algorithms in order to obtain a contour of the object. The object contour is a base for different geometrical measurements (Klette and Rosenfeld [1]).

The simplest way to determine the contour length is counting the pixels on the detected contour with the 4-connected or 8-connected method (Klette and Rosenfeld [1]). The 4-connected method enables counting the vertical and horizontal elements while the 8-connected method additionally counts the diagonal elements. In both methods the counts of horizontal, vertical or diagonal elements are multiplied with the distance between two centre points of the particular element and the approximate length is obtained. Both methods enable only a rough approximation of the real object length. If other directions besides those three elementary ones are taken into account determined by the image grey level gradient, more accurate results can be achieved (Eberly and Lancaster [2]).

In general, summing up distances between consecutive pixel centres is not accurate enough to determine a digital curve length. In order to measure the object's length more accurately, several other methods have been developed. Besides the methods with different line length estimators (Dorst and Smeulders [3], Vossepoel and Smeulders [4]), more sophisticated methods with the straight line segments extraction (Braquelaire and Vialard [5], Klette and Yip [6]) or calculation of the shortest path through the polygon (Sloboda et al. [7]) have been tried out as well. Recently, an active contour model (Peng and Zhao-Bang [8]) and a model with a spline approximation (Foteinopoulos [9]) have also been implemented in the case of perimeter measurement of the planar objects.

The authors of this contribution have so far developed the method of anchored discrete convolution with the rectangular, triangular and binomial masks (Suhadolnik et al. [10]). To test its reliability, we will use here a Bézier convolution mask and compare it to the results obtained with the binomial mask. The length calculations obtained by both masks will be implemented to real samples obtained by an optical scanner.

\section{Digital curve and arc length}

In the following calculation, the digital image will be represented as a grid of $M \times N$ square pixels measuring $2 \Delta$ in both directions. An edge detecting algorithm applied to the digital image extracts the object's contour (Castelman [11]) which can be represented by a sequence of $n$ consecutive pixels $P_{0}, P_{1}, P_{2}, \ldots, P_{i}, \ldots, P_{n-1}$. These consecutive pixels have at least one common edge or corner. If the path through the consecutive pixels is closed, then the pixels represent a digital curve $\Gamma_{C}$ (fig. 1).

The length $\Delta_{i}$ between two consecutive pixels centres $\ldots . \mathbf{P}_{i}, \mathbf{P}_{i+1}, \ldots$. in the pixels $\ldots . . P_{i}, P_{i+1}, \ldots$ of the digital curve $\Gamma_{C}$ is $\Delta_{i}=\left|\mathbf{P}_{i+1}-\mathbf{P}_{i}\right|$. The complete approximate digital curve length which represents a closed path can be calculated by summing up all distances between the pixels centres: 


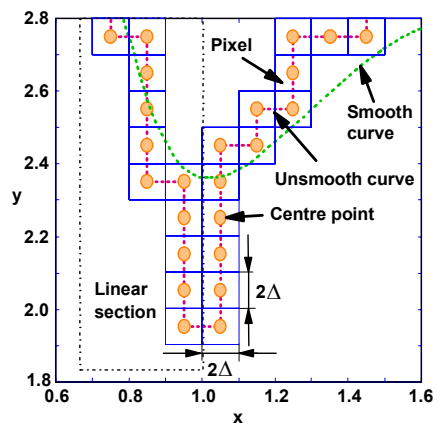

Figure 1: $\quad$ Part of digital curve $\Gamma_{C}$.

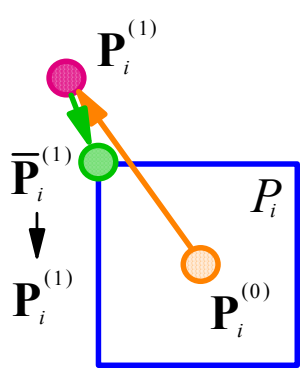

Figure 2: $\quad$ Pixel with anchoring.

$$
L_{C}=\left|\mathbf{P}_{n-1}-\mathbf{P}_{0}\right|+\sum_{i=0}^{i=n-2}\left|\mathbf{P}_{i+1}-\mathbf{P}_{i}\right|
$$

\section{Anchored discrete convolution}

The initial pixels centre points are denoted by $\mathbf{P}_{i}^{(0)}=\mathbf{P}_{i}, i=0,1,2, \ldots, n-1$. After the discrete convolution calculation the centre points $\mathbf{P}_{i}^{(0)}$ move from the initial positions and the moved points $\mathbf{P}_{i}^{(l)}$ are achieved, where $l$ denotes the number of the consecutive iterations throughout the rest of the text (fig. 2). On the closed curve $\Gamma_{C}$ the following identities hold: $\mathbf{P}_{n-1}=\mathbf{P}_{-1}, \mathbf{P}_{n}=\mathbf{P}_{0}$ and $\mathbf{P}_{n+1}=\mathbf{P}_{1}$.

\subsection{Discrete convolution with the binomial mask}

If $G_{j}$ denotes the convolution mask of order $k$, then the discrete convolution equation which influences $n$ vertices is equal to (Suhadolnik et al. [10]):

$$
\mathbf{P}_{i}^{(l+1)}=\sum_{j=-k}^{j=k} G_{j} \mathbf{P}_{i+j}^{(l)}, \quad i=0,1,2 \ldots, n-1 .
$$

For the binomial mask $G_{j}$ is equal to (fig. 3a):

$$
G_{j}=\frac{1}{2^{2 k}}\left(\begin{array}{c}
2 k \\
k+j
\end{array}\right), \quad j=-k,-k+1, \ldots \ldots ., k .
$$

The convolution mask $G_{j}$ has normalized value $\sum_{j=-k}^{j=k} G_{j}=1$. 


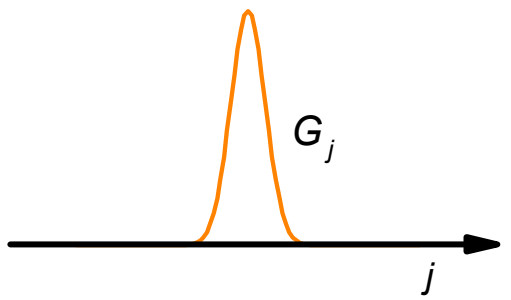

a) Binomial mask.

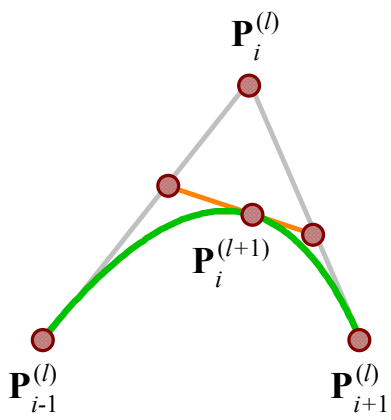

b) Bézier second order convolution.

Figure 3: Discrete convolution.

\subsection{Convolution based on marker points of the second order Bézier curve}

Let us define three consecutive points $\mathbf{P}_{i-1}^{(l)}, \mathbf{P}_{i}^{(l)}$ and $\mathbf{P}_{i+1}^{(l)}$ as the Bézier marker points. With the Bézier curve of the second order one can calculate $\mathbf{P}_{i}^{(l+1)}$ according to the following equation (Farin [12]):

$$
\mathbf{P}_{i}^{(l+1)}=\left(1-\lambda_{i}^{(l)}\right)^{2} \mathbf{P}_{i-1}^{(l)}+2 \lambda_{i}^{(l)}\left(1-\lambda_{i}^{(l)}\right) \mathbf{P}_{i}^{(l)}+\lambda_{i}^{(l) 2} \mathbf{P}_{i+1}^{(l)},
$$

where $i=0,1,2, \ldots, n-1$. If the parameter $\lambda_{i}^{(l)}$ has values between $0 \leq \lambda_{i}^{(l)} \leq 1$ then the point $\mathbf{P}_{i}^{(l+1)}$ lies within the convex hull which is formed by the Bézier marker points (fig. 3b). The parameter $\lambda_{i}^{(l)}$ is defined as a length ratio:

$$
\lambda_{i}^{(l)}=\frac{\left|\mathbf{P}_{i}^{(l)}-\mathbf{P}_{i-1}^{(l)}\right|}{\left|\mathbf{P}_{i}^{(l)}-\mathbf{P}_{i-1}^{(l)}\right|+\left|\mathbf{P}_{i+1}^{(l)}-\mathbf{P}_{i}^{(l)}\right|}=\frac{\Delta_{i-1}^{(l)}}{\Delta_{i-1}^{(l)}+\Delta_{i}^{(l)}},
$$

\subsection{Anchoring discrete convolution algorithm}

If the convolution is applied on the initial centre points the curve becomes smoother by lowering the curve peaks (fig. 1). Smoothing introduces distortions on the original curve which loses its initial shape. Large points shifting are prevented by anchoring the points within the pixels.

In the case of the Bézier convolution mask, the length calculation is performed in several iteration steps, while in the case of binomial mask both the iterative calculation and calculation in one single step are used. In the iterative way the calculation steps are repeated until the difference between two consecutive length results is less than a predefined small $\varepsilon$. At the beginning of each calculation the points are in the centre positions in the pixels $\mathbf{P}_{i}^{(0)}=\mathbf{P}_{i}$. The derived points denoted by $\mathbf{P}_{i}^{(l+1)}$ are displaced from the previous positions $\mathbf{P}_{i}^{(l)}$ 
during the calculation. If the derived point $\mathbf{P}_{i}^{(l+1)}$ is out of the pixel $\left(\mathbf{P}_{i}^{(l+1)} \notin P_{i}\right)$, an anchoring is applied as shown in figure 2 and the point is moved onto the pixel border. New point is denoted by $\overline{\mathbf{P}}_{i}^{(l+1)}$ and after relocation on the pixel border treated as a regular point $\overline{\mathbf{P}}_{i}^{(l+1)} \rightarrow \mathbf{P}_{i}^{(l+1)}$ in the next iteration step. The anchoring convolution keeps the points within the pixels during iterative calculation $\mathbf{P}_{i}^{(l)} \in P_{i}$. The approximate close curve length in each iterative step $l$ is then:

$$
L_{C}^{(l)}=\left|\mathbf{P}_{n-1}^{(l)}-\mathbf{P}_{0}^{(l)}\right|+\sum_{i=0}^{i=n-2}\left|\mathbf{P}_{i+1}^{(l)}-\mathbf{P}_{i}^{(l)}\right| .
$$

The iterative calculation is finished when the condition $\left|L_{C}^{(l)}-L_{C}^{(l-1)}\right|<\varepsilon$ is fulfilled for a predefined small $\varepsilon$. The last result is the approximate digital curve length. In the case of the binomial mask, the convolutions order $k=4$ is used.

\section{Length measurements}

The anchored convolution algorithm was tested on calculating the length of an object in digital images. The first measurement was performed on an ideal circle with a known radius. In figure 4 the calculated curves with the Bézier mask and the binomial mask of order $k=4$ are shown. The extents of both curves are in excellent agreement with the circle circumference of 6.5596. The Bézier mask gives the extent length of 6.5532 and the binomial mask without iterations 6.5200 .

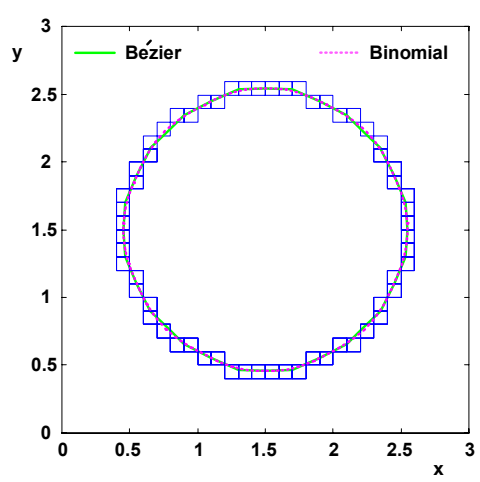

Figure 4: Circle extent measurement.
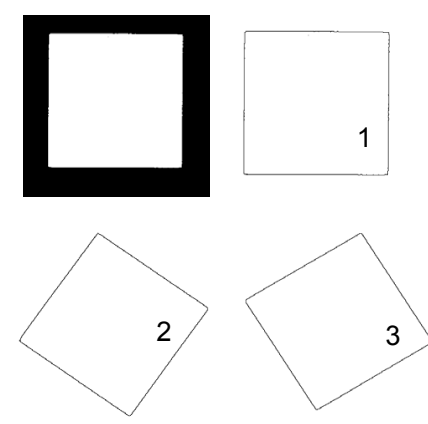

Figure 5: Scanned square object and outline of the square with different rotations.

Further measurements were performed on a square sample. The object was scanned with an optical scanner at different resolutions of 75, 150, 300 and 
600 dpi. The square was scanned at three different rotations (fig. 5) and the outline of the object was determined after each scan. The acquired outline of the object was transformed into a chain code (Castelman [11]). The 4-connected and 8 -connected chain codes were used and the achieved results are presented in table 1 .

Table 1: $\quad$ Square perimeter measurement.

\begin{tabular}{|c|c|c|c|c|c|c|}
\hline \multirow[b]{3}{*}{ DPI } & \multicolumn{6}{|c|}{ Square perimeter in $\mathrm{mm}$ (real value $=97,22 \mathrm{~mm}$ ) } \\
\hline & \multicolumn{2}{|c|}{$\begin{array}{c}\text { Bézier } \\
\text { (iterative) }\end{array}$} & \multicolumn{2}{|c|}{$\begin{array}{l}\text { Binomial } \\
\text { (iterative) }\end{array}$} & \multicolumn{2}{|c|}{$\begin{array}{c}\text { Binomial without } \\
\text { iterations }\end{array}$} \\
\hline & $4^{*}$ & $8^{*}$ & $4^{*}$ & $8^{*}$ & $4^{*}$ & $8^{*}$ \\
\hline \multicolumn{7}{|c|}{ Square rotation first position (1) } \\
\hline 75 & 98,03 & 97,66 & 97,22 & 97,15 & 97,48 & 97,50 \\
\hline 150 & 97,10 & 97,26 & 96,98 & 96,93 & 97,14 & 97,13 \\
\hline 300 & 96,85 & 97,01 & 96,70 & 96,72 & 96,96 & 96,90 \\
\hline 600 & 97,03 & 97,18 & 96,61 & 96,60 & 97,20 & 97,01 \\
\hline \multicolumn{7}{|c|}{ Square rotation second position (2) } \\
\hline 75 & 98,66 & 97,75 & 97,04 & 97,22 & 97,61 & 97,44 \\
\hline 150 & 98,09 & 97,07 & 96,56 & 96,65 & 97,05 & 96,83 \\
\hline 300 & 98,59 & 97,03 & 96,62 & 96,67 & 97,09 & 96,78 \\
\hline 600 & 98,59 & 97,04 & 96,67 & 96,61 & 97,19 & 96,76 \\
\hline \multicolumn{7}{|c|}{ Square rotation third position (3) } \\
\hline 75 & 99,03 & 97,95 & 97,10 & 97,25 & 97,65 & 97,55 \\
\hline 150 & 97,83 & 97,30 & 96,62 & 96,72 & 97,05 & 96,90 \\
\hline 300 & 98,30 & 97,10 & 96,55 & 96,57 & 96,93 & 96,70 \\
\hline 600 & 98,53 & 97,18 & 96,67 & 96,63 & 97,15 & 96,78 \\
\hline
\end{tabular}

( $4 *-4$ connected method, $8 *-8$ connected method.)

Table 1 shows that the Bézier mask of second order gives a better result in the 8 -connected case than in the 4-connected case, while the differences in the results of the binomial mask in both cases are negligible. The 4-connected and 8connected digital lines at different slopes and approximate curves are shown in fig. 6. Furthermore the rotation of the square object has no significant influence on the measured results. This is an important feature which favours the use of the anchored convolution in digital image measurements. It is also observed from table 1 and fig. 6 that in the case of the binomial mask the procedure in one step without iterations gives a better result than the iterative procedure. The results in the iterative procedure are constantly lower than the real value (for more information see [10] by Suhadolnik et al). The iterative approach shrinks the curve at the corners toward the inner border. The calculation without iterations is fast and introduces fewer distortions. On the contrary, the Bézier mask gives inaccurate results without iterative calculation. 


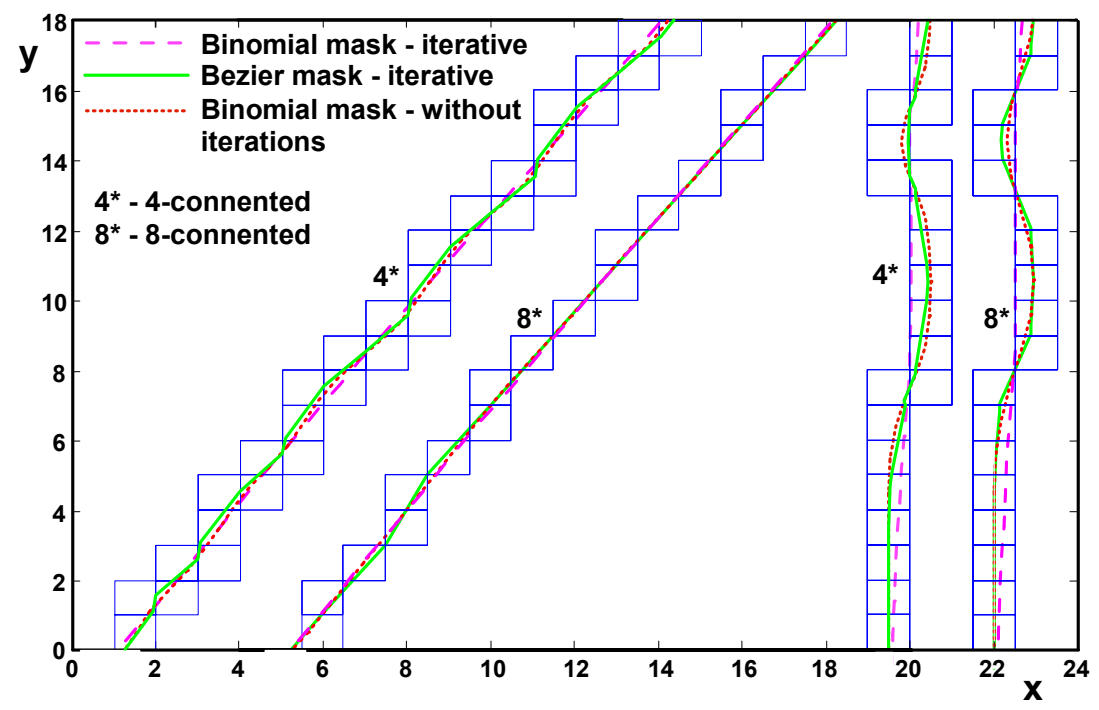

Figure 6: Digital lines at different slopes and approximate curves.

In figure 7 additional scanned objects at 600 dpi with the 8 -connected contours are shown and their outer extents are calculated. In the case of the key the extent length is $148.94 \mathrm{~mm}$ with the Bézier convolution mask and 147.91 $\mathrm{mm}$ with the binomial mask without iteration, and in case of the puzzle piece the extent length is $120.32 \mathrm{~mm}$ and $119.45 \mathrm{~mm}$ for the same masks.

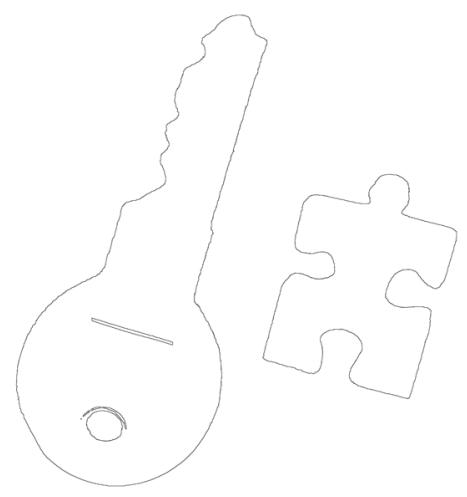

Figure 7: Outlines of the key and the puzzle piece.

\section{Conclusions}

The paper presents the anchored discrete convolution method for the determination of an object length in a digital image. The calculated points are 
derived by using the binomial convolution mask and the mask based on the marker points of the second order Bézier curve. In both cases the large point shifting is prevented by anchoring the points into the pixels. Good results are achieved by the iteration procedure with the Bézier mask and without iterations with the binomial mask. To verify this finding, some scanned objects are measured and the results are presented.

\section{References}

[1] Klette R. \& Rosenfeld A., Digital geometry, Elsevier: Singapore, 2006.

[2] Eberly D. \& Lancaster J., On gray scale image measurements. Graphical Models and Image Processing, 53(6), pp. 538-549, 1991.

[3] Dorst L. \& Smeulders A.W.M., Length estimators for digitized contours. CVGIP, 40(3), pp. 311-333, 1987.

[4] Vossepoel A.M. \& Smeulders A.W.M., Vector code probability and metrication error in the representation of straight lines of finite length. Computer Graphics and Image Processing, 20(4), pp. 347-364, 1982.

[5] Braquelaire J-P. \& Vialard A., Euclidean Paths: A New Representation of Boundary of Discrete Regions. Graphical Models and Image Processing, 61(1), pp. 16-43, 1999.

[6] Klette R. \& Yip B., The Length of Digital Curves. Machine Graphics \& Vision, 9(3), pp. 673-703, 2000.

[7] Sloboda F., Zatko B. \& Stoer J., On approximation of planar onedimensional continua. Advances in Digital and Computational Geometry, eds. R. Klette, A. Rosenfeld \& F. Sloboda, Springer Verlag: Singapore, pp. 113-160, 1998.

[8] Peng Z. \& Zhao-Bang P., Precise perimeter measurement for planar object. Measurement, 41(8), pp. 878-884, 2008.

[9] Foteinopoulos P., Cubic spline interpolation to develop contours of large reservoirs and evaluate area and volume. Advances in Engineering Software, 40(1), pp. 23-29, 2009.

[10] Suhadolnik A., Petrišič J. \& Kosel F., Numerical calculation of digital curve length by using anchored discrete convolution. Image and Vision Computing, 26(7), pp. 990-999, 2008.

[11] Castelman K., Digital Image Processing, Prentice-Hall: New Jersey, 1979.

[12] Farin G., Curves and Surfaces for CAGD, A Practical Guide, Academic Press: San Diego, 2002. 